\title{
Tocainide Hydrochloride
}

National Cancer Institute

\section{Source}

National Cancer Institute. Tocainide Hydrochloride. NCI Thesaurus. Code C47761.

The hydrochloride salt form of tocainide, a primary amine analog of lidocaine exhibiting class $1 \mathrm{~b}$ antiarrhythmic property. Tocainide hydrochloride stabilizes the neuronal membrane by reversibly binding to and blocking open and inactivated voltage-gated sodium channels. This inhibits the inward sodium current required for the initiation and conduction of impulses and reduces the excitability of myocardial cells. This agent reduces the rate of rise and amplitude, and shortens the action-potential duration (APD) in both the Purkinje and muscle fibers. Tocainide also shortens the effective refractory period (ERP) of Purkinje fibers resulting in an increased the ERP/APD ratio. Overall these effects lead to the slowing of nerve impulses and stabilization of the heartbeat. 